\title{
TOWARDS MACHINE VISION BASED RAILWAY ASSETS PREDICTIVE MAINTENANCE
}

\author{
$U D C(621.3: 629.056 .84)$
}

\section{Ivan Ćirić, Milan Banić, Miloš Simonović, Aleksandar Miltenović, Dušan Stamenković, Vlastimir Nikolić}

\author{
University of Niš, Faculty of Mechanical Engineering, Niš, Republic of Serbia
}

\begin{abstract}
The main goal of this paper is to present novel technologies that can contribute to safety, competitiveness, efficiency and operational reliability of Railway infrastructure through the development of innovative solutions for measuring and monitoring of railway assets based on machine vision. Measuring the transversal position of the wheels on the rail, as well as identification of the defects of the wheel and the rail (such as deformation of rail head edge, lateral wear, worn wheels, cracks in wheel and rail, rolling contact fatigue, corrugation and other irregularities) can increase reliability and lower maintenance costs. Currently, there is a need on the market for the innovative solution, namely the onboard high-speed stereo camera system augmented with a system that projects custom pattern (fringe scanner system) for measuring the transversal position of the wheels on the rail, robust to environmental conditions and waste along the track that can provide reliable measurements of transversal position of the wheels up to $200 \mathrm{~km} / \mathrm{h}$. New trends in Precise Industrial 3D Metrology are showing that stereo vision is an absolute must have in modern specialized optical precision measuring systems for the three-dimensional coordinate measurement.
\end{abstract}

Key words: machine vision, stereo vision, predictive maintenance, wheel-rail contact

\section{INTRODUCTION}

One of the ways to increase the efficiency and operational reliability of the existing Railway infrastructure is to automate the inspection activities as well to increase the unification and increase the monitoring and measuring scope of systems currently available. Visual inspections of vehicles and infrastructure are currently preformed manually by the maintenance staff which is a time and cost consuming process. Current measuring and monitoring solutions focus predominately on determination of specific set of defects and their quantification which aggravates the fusion of measurement data for the vehicle as a whole, or

Received August 29, 2020

Corresponding author: Ivan Ćirić

University of Niš, Faculty of Mechanical Engineering, Aleksandra Medvedeva 14, 18000 Niš, Republic of Serbia

E-mail: ciric.ivan@masfak.ni.ac.rs 
for the infrastructure as a system. Furthermore, the effects of rolling stock defects on the infrastructure are not clearly established which hampers the infrastructure maintenance planning $[1,2]$.

In order to maintain competitiveness with other means of transport it is necessary to accept Industry 4.0 trends and advances in the terms of gathering more data for better predictive maintenance planning as a component of a larger asset management strategy to diagnose, prevent and postpone failures. Such an approach lowers the number of service disruptions, enables the timely preparation of parts and maintenance staff and decreases costs over routine or time-based preventive maintenance because maintenance activities are performed only when necessary. The definition of models which quantify the impact of measured defects to the infrastructure enables the shift to proactive maintenance as the root causes of infrastructure wear and failure can be predicted and eliminated before any measurable damage occurs. Monitoring wheelsets and particularly wheels in service significantly increases the reliability and reduces maintenance costs. Also, due to regulations (UIC 660, 2002) to allow power and speed to be reduced in the event of instability, high-speed rolling stock must be fitted with an approved system for detecting the bogie instability on a permanent basis. It is acceptable for the system to be activated only when running at speeds higher than $220 \mathrm{~km} / \mathrm{h}$ (i.e. for high/speed operations).

It is one of the key requirements to enable the calculation of fatigue and wear levels of the wheel and the rail. Better prediction to the wheel and rail fatigue and wear enable the predictive maintenance of the noted components as the most important component of a railway system. A better control of wheel and rail wear also leads to a decrease of overall dynamic forces which act on the rolling stock thus increasing their service life as well as decreasing the costs related to maintenance.

Railway wheels sit on the rails without guidance except for the shape of the tyre in relation to the rail head. Contrary to the popular belief, the flanges should not touch the rails. Flanges are only a last resort to prevent the wheels becoming derailed - they are a safety feature. The wheel tyre is coned and the rail head slightly curved as shown in the following diagram (Figure 1a) [1].
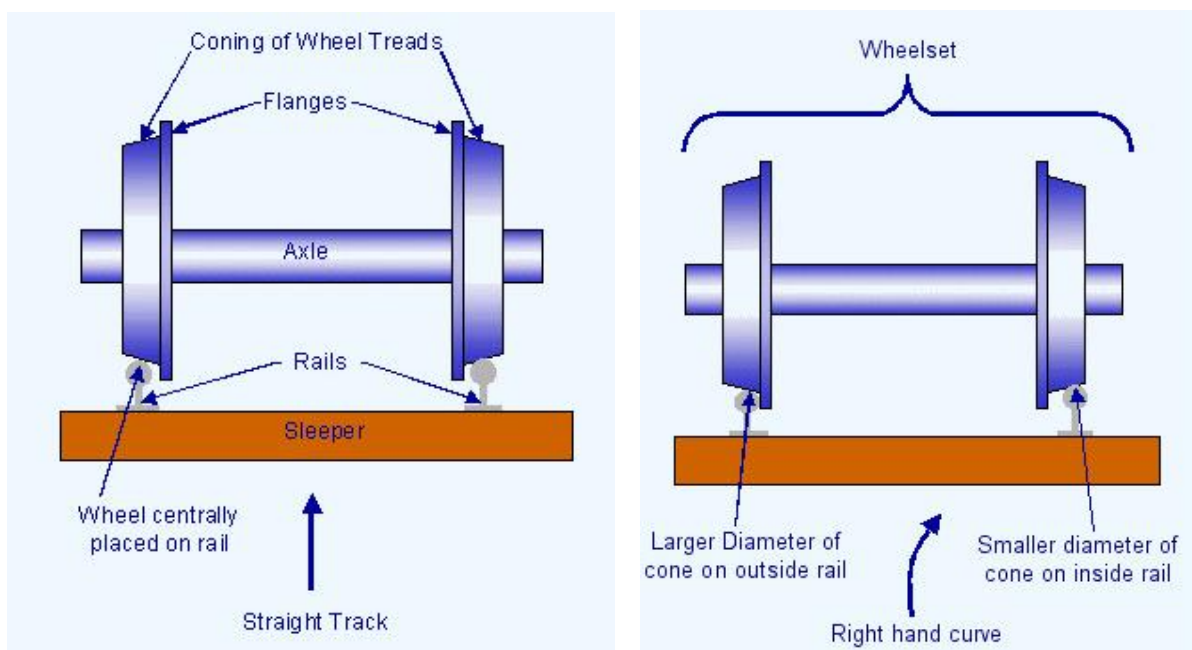

Fig. 1 The shape and location of wheels and rails on straight track (a) and location of the wheels in relation to the rails on curved track (b) 
This diagram is exaggerated to show the principle of the wheel/rail interface on a straight track. Note that the flanges do not normally touch the rails.

The motion of the wheel on the rail on the straight track is called a "sinusoidal ride". This is presented in Figure 2 [1].

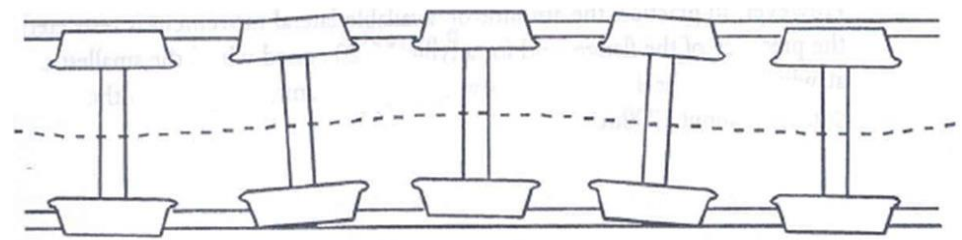

Fig. 2 Sinusoidal motion of wheelset on straight track

On a curved track, the outer wheel has a greater distance to travel than the inner wheel. To compensate for this, the wheelset moves sideways in relation to the track so that the larger tyre radius on the wheel cone is used on the outer rail of the curve, as shown in Figure1-b.

The inner wheel uses the outer edge of its tyre to reduce the travelled distance during the passage round the curve. The flange of the outer wheel will only touch the rail if the movement of the train round the curved rail is not in exact symmetry with the geometry of the track. This can occur due to the incorrect speed or poor mechanical condition of the track or train.

There will always be some slippage between the wheel and rail on curves, but this will be minimised if the track and wheel are both constructed and maintained according to the prescribed regulations.

\section{MEASUREMENT OF THE WHEEL-RAIL CONTACT}

Precise measurement of the transversal position of the wheel in relation to the rail can lead to a better prediction of the wheel and rail fatigue and wear. These predictions are enabling the predictive maintenance of the noted components as the most important component of a railway system. A better control of the wheel and rail wear is also leading towards decreasing of overall dynamic forces which act on the rolling stock thus increasing their service life as well as decreasing the costs related to the maintenance.

Researchers were mainly dealing with problems considering wheel/rail contact forces.

Matsumoto et. al. [1] have presented a new measuring method for the wheel-rail contact forces that can measure contact forces without special wheelsets equipped with strain gauges and slip rings or telemeters. In this method the lateral contact force is measured from the lateral distortion of a wheel measured directly by several non-contact gap sensors. Normal and longitudinal contact forces are also measured from the deflection of spring or the strain of parts of the bogies except wheelsets.

In [2] non-contact gap sensors were equipped on non-rotating parts of a bogie, and a new measuring method of wheel/rail contact forces has been developed. The developed system has been verified to have the sufficient durability for continuous measurement on 
in-service trains and the sufficient practical accuracy after various stand tests and train running tests. After a long-period of continuous measurement on a commercial subway line, some important characteristics of wheel/rail contact mechanics were found by the analysis of measured data.

Wheel/rail contact forces of high-speed train are very difficult to measure directly and some researchers are dealing with the estimation of this force. Zhu et al. [3] a timedomain inversion method for dynamic loads was proposed. Based on the state space equation, dynamic programming methods and the Bellman principle of optimality, the main theoretical derivation of the inversion mathematical model was given. With a highspeed vehicle system as the research object, accelerations of axle box as input conditions, the vertical and horizontal wheel/rail forces were identified.

This contact force was estimated by Wei et al. [4] by the measurement of the wheel set flange derailment. An indirect method for wheel/rail force measurement was proposed in this paper, while comparison of the force time history applied to the instrumented wheel set and that obtained using the indirect method is presented.

Bagheri et al. [5] are dealing with optimal strain gauge placement in an instrumented wheelset for measuring wheel-rail contact forces. For measurement of the contact forces an instrumented wheelset has been developed and a novel technique for precise design of the instrumented wheelset is presented in this paper. Influences of various factors such as the wheel rotation, the temperature or the centrifugal force are eliminated by cancelation of the higher harmonics while the accuracy of estimated forces is dependent on the placement of gauges on the instrumented wheelset.

The system for assessment of the wheel-rail contact forces can be used for multiple purposes, among them for estimation of the train running safety, for train axle load measuring or wheel flats detection, as well as for the other research analyses. As the wheelrail rolling contact moves along the track during train motion, it is very difficult to establish a reliable and accurate system for measuring of contact forces. The measurement principle of the wayside system, presented by Milkovic et al. [6] is based on rail strains measurements using strain gauges, connected into the Wheatstone bridges in a smart way, in order to achieve a signal proportional to the applied load. This principle uses an independent component analysis (ICA) model in combination with the system calibration for the successful separation of vertical and lateral contact forces from the recorded strain signals. In addition, the system provides identification of the contact point position on the rail during train passing over it, which further expands the possibilities of its application to wheel-rail wear analysis, contact geometry optimization etc.

Introducing the measurement of the dynamic change of $t$ he transversal position of the wheel in relation to the rail into the simulation model used for wheel/rail force estimation can improve the estimator performance. Since all the researchers dealing with the wheel and rail contact forces agree that a continuous measurement of these forces is extremely complicated, every feasible solution is based on some sort of estimation. The estimation of these forces is done by a complex simulation of rail and wheel dynamics, while some other forces and parameters are measured. The measured transversal position can be introduced either in a complex mathematical model, or in a simulation model in the specialized simulation software. This addition to the mathematical/simulation model can for sure improve the simulation accuracy and therefore the estimator performance.

For measurement of the wheel-rail contact there are also on-track solutions used just for the wheel profile inspection. The interaction between wheels and rails is one of the 
key factors affecting the dynamical behavior of rolling stock, and consequently, the safety and comfort of railway vehicles, so it is necessary to detect with high accuracy and frequency the different parameters of the wheels, and in particular the contact profile. The DWPM, DANOBAT's latest generation of Wheel Profile Measuring Systems, is used for on-track wheel profile inspection system.


Fig. 3 TTCI WRCI system [7]

TTCI (Transportation Technology Center, Inc.) has developed an automated wheel/rail contact inspection (WRCI TM) system (Figure 3) using the Track Loading Vehicle or existing track geometry measurement cars (Wu 2006). A laser system installed in front of the leading truck of TTCI's Track Loading Vehicle is used to measure the dynamic rail profiles. The rail profiles are measured as the car moves along the track. TTCI's approach is to match measured rail pairs against wheels typical of the vehicles passing over, calculate the wheel/rail interaction, and assess against the preferred interaction. The data analysis software computes the contact parameters by placing each wheelset on the measured rail pair. The maximum operating speed for real-time data analysis can now reach up to $47 \mathrm{mph}(75 \mathrm{~km} / \mathrm{h})$ with a processing interval of 10 feet (3 m) by using multiple data processors.

The rail/wheel contact measurement system determines (1) Contact angle-indication of flange climb risk, (2) Low rail contact position-indication of rail rollover, (3) High rail contact conformity-indication of bogie steering, (4) Conicity-indication of high speed lateral stability, (5) Rolling radius difference-indication of bogie steering, and (6) Contact stress-indication of rolling contact fatigue risk.

The company Deutzer Tehnische Kohle (DTK, [8]) is offering Wheel-Rail contact measurement system as a product (Figure 4).

The system for detection of the wheels position to the rail is adaptable to customers' specific needs. It is used for measurement of the distance between the wheel and the rail. All measurement data are synchronized with videos and Audio comments at salient way points and as a result customer can have a detailed measurement report.

The distance measuring results are very accurate when measuring wheel and rail contact with only one laser unit. Points where the wheel is climbing up the rail can be detected very easily to avoid derailing. Optionally the track geometry and the rail profile 
can be recorded, exact coordinates for every position with GPS or dGPS can be recorded. The system provides distance between the Wheel and the Rail for both sides.

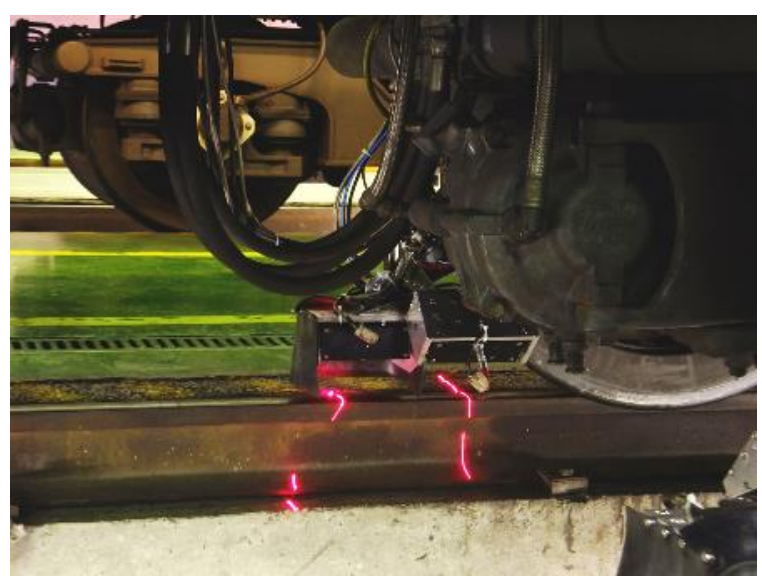

Fig. 4 DTK Wheel-Rail contact measurement system [8]

The DTK track geometry measurement system is another commercial product of the same company that logs the entire track geometry and track profiles for regular rail services. Variables captured during measurement are gauge, groove width, groove depth or railhead height, current contour of rails (wear), track cant, twisting, yaw angle, ramps, longitudinal level, alignment, curve radius, shocks between wheel and rail, smooth running of axle on the rails (loosening of rails), position on the route (GPS coordinates and route) and speed. Optionally, this system can include the Rail-wheel contact. The track geometry is obtained under load on the standard passenger rolling stock of the relevant transport operator. Even while still on the measurement journey, a first impression can be obtained of the platform geometry since the measurement data is displayed on monitors. After a second measurement the measurement campaigns can be compared to one another to determine changes in the track geometry or in the contours. This provides information on how the wear of the infrastructure has developed. DTK evaluation software enables customer to determine all deviations above and below the specified measurement tolerances. To evaluate wear, for each chosen route position the current contour of the rails can be displayed and compared with the ideal contour.

BVSYS [9] as a commercial product has a specialized measuring system 3rdRailPositionCheck. This measuring system allows the real-time, automatic measurement of the lateral position and height of the contact rail relative to the side rail. 3rdRailPositionCheck is an automatic real-time measuring system to determine the position of contact rails on tracks. Measurement reports are generated as a result that shows the deviations of the contact rail's position from the set position and generate an error message if tolerances are exceeded. In the basic version, the system works up to a speed of $130 \mathrm{~km} / \mathrm{h}$ at a measurement interval of $10 \mathrm{~cm}$, while higher speeds and shorter intervals are possible depending on the requirements.

The measuring system is based on the split-beam method and in each case consists of a line laser and a camera. A laser line projected onto the rail is recorded by means of a special 
digital camera. Powerful computers automatically evaluate the image data and generate the reference points necessary for the relevant position. Using these reference points, which are generated by four sensor units, the position of the 3rd rail is calculated relative to the side rail. Figure 5 shows the principle of measurement, while Figure 6 shows the measured values by the 3rdRailPositionCheck.

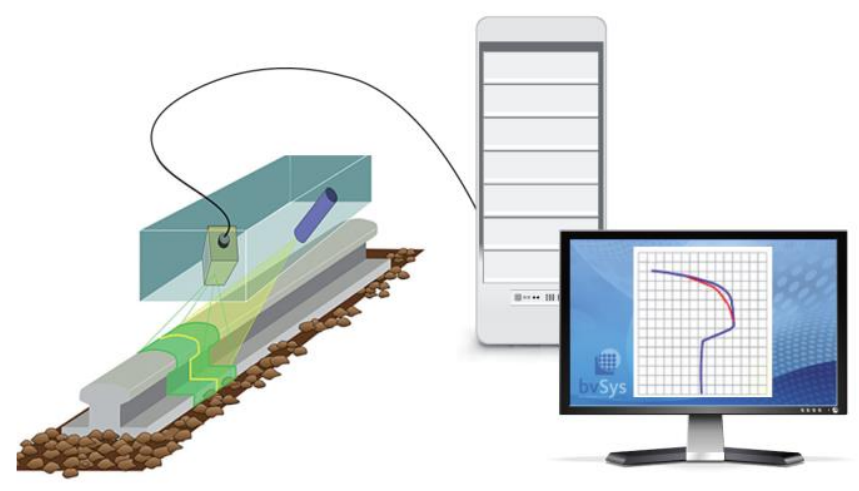

Fig. 5 Principle of measurement by BVSYS 3rdRailPositionCheck [9]

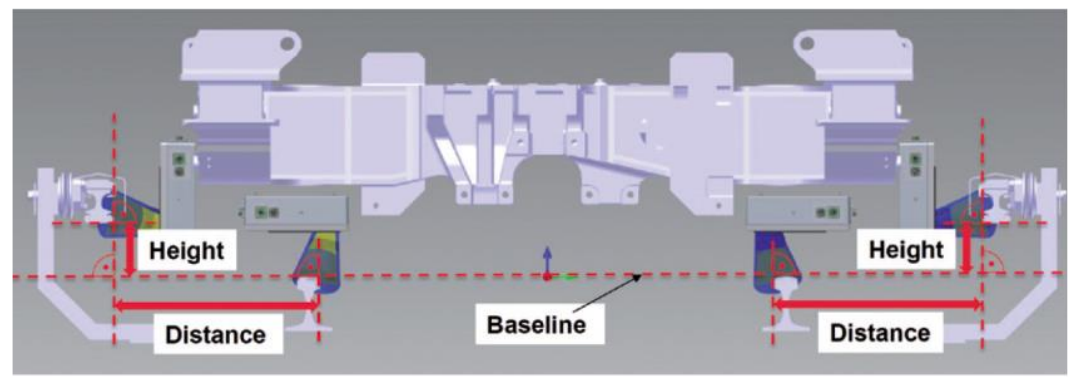

Fig. 6 Measured Values by BVSYS 3rdRailPositionCheck [9]

There is another BVSYS product ProfileCheck, a high-precision optical measurement system for the contactless inspection of vertical and cross-sections of railway tracks at a speed of over $200 \mathrm{~km} / \mathrm{h}$ (Figure 7). The system is used for the early detection of rail damage in areas of the running edge and rail surface thereby enabling reprofiling of the rails at an early stage, which increases the lifetime considerably.

The system is based on the measuring principle of contactless laser triangulation. Thanks to a new, patented method, the measurement data is detected especially during the vertical section measurement independent of movements of the sensor in relation to the rail. As a result of this, short and long waves can be measured on the track with the highest precision and quantified with regard to the amplitude part. The cross-section measuring system is used for the precise measurement of the rail head as well as the gauge. The system is able to record and evaluate a complete rail cross-section every $0.096 \mathrm{~m}$ at a speed of $160 \mathrm{~km} / \mathrm{h}$. During the inspection run, the gauge, the amplitudes of 
the corrugation and waves as well as the rail head section are displayed visually online. In the fault protocol that is created, faulty track areas are identified to the exact metre by means of the track correlation through the chainage that runs in parallel.

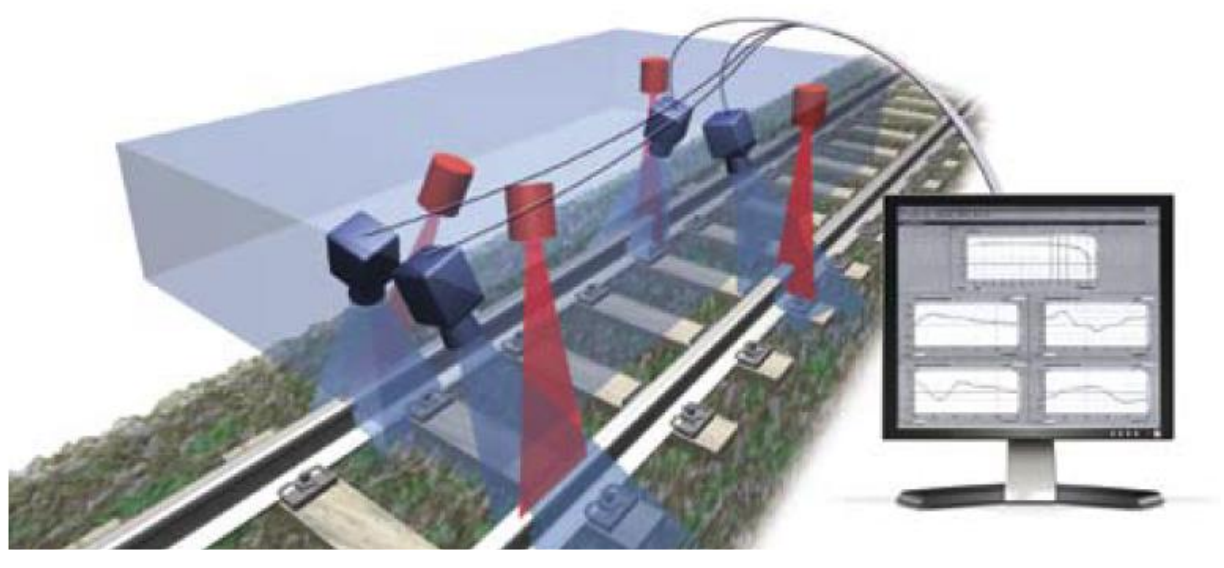

Fig. 7 BVSYS ProfileCheck operating principle [10]

The system is scalable to a speed of up to $320 \mathrm{~km} / \mathrm{h}$, it has high measuring precision, it is easily operable with an intuitive controllable user interface. The modular concept of the measuring system allows low maintenance cost, easy integration thanks to small space requirements both under and in the vehicle. It is an open system; thereby easy for adaption to country-specific database systems or central data acquisition on the vehicles.

\section{MACHINE Vision in Reliable WhEEL-RaIL CONTACT MEASUREMENT}

The on-board system for measuring the transversal position of the wheels on the rail can be based on the high-speed camera pair (stereo pair) augmented with the pattern projecting technology (fringe scanner system). Grid/pattern projected onto the wheel/rail interface can then be recorded by means of a high-speed stereo camera system. Stereo image data should be processed by an integrated computer to reconstruct the 3D point cloud of the wheel/rail interface and generate the reference measurement points for the determination of the transversal position of the wheel on to the rail. Such a solution with a GPU accelerated low power system board, which will enable the sensor connection and processing, can provide reliable measurements of the transversal position of the wheels. The application of a high speed camera with up to 5000 FPS will enable the system functioning even at speeds greater than $200 \mathrm{~km} / \mathrm{h}$. As the system is primarily based on vision sensors, aside of its primary function (measuring the transversal position of the wheels on the rail) it can be able to identify the defects of the wheel and the rail such as the deformation of the rail head edge, lateral wear, worn wheels, cracks in wheel and rail, rolling contact fatigue, corrugation and other irregularities.

New trends in Precise Industrial 3D Metrology are showing that the stereo vision is an absolute must have in modern specialized optical precision measuring systems (www.gom.com) for the three-dimensional coordinate measurement. With high speed 
cameras with the frame rate up to 20000 (http://www.phantomhighspeed.com/, V2640) and GPU accelerated low power board, precise measurement and 3D reconstruction is possible for speed of the moving train much higher than $200 \mathrm{~km} / \mathrm{h}$.

When considering stereo-vision based systems, two cameras, displaced horizontally from one another are used to obtain two differing views on a scene, in a manner similar to the human binocular vision. In general, two types of methods are used to interpret the data obtained from stereo vision sensors in driver assistant systems:

- Disparity maps

- Inverse Perspective Mapping (IPM)

Although computation of a disparity map is time consuming, Mandelbaum et al. [11] shows that it can be done real-time. A review on different methods of depth estimation using stereo camera system can be found in [12]. In general, in disparity maps, positions of pixels on both cameras that represent the same object are compared. The third component of the pixel vector ( $\mathrm{z}$ coordinate) can be calculated by comparing changes in pixels position, while the inverse perspective mapping method is based on transforming the image and removing the perspective effect from it [13]. An early example of applying the inverse perspective mapping can be found in (Zhao et al. 1993), where the contours of the objects which stand above the ground are extracted from the images.

Fraunhofer Institute for Applied Optics and Precision Engineering has developed a High-speed 3D-measurement system with gobo-projection [14]. It is a touchless optical 3D measurement system with a triangulation-based 3D measurement. Stereo vision-based image acquisition using high-speed cameras and high-speed projection of aperiodic fringe patterns enables 3D measurements of highly dynamic situations and large measurement fields. The measuring principle with Gobo (Graphical optical blackout)-based high-speed Projection of fringe patterns with the use of high-speed cameras allows to record up to $10,0003 \mathrm{D}$ images /s. There are many papers dealing with the 3D stereo measurement with projection patterns $[15,16,17,18,19]$.

One of the needs for adequate functionality of the measurement of transversal position of wheel in relation to the rail system is insensitive to dust, rain, snow and ice. Housing IP68 protection will provide an adequate protection of components, but for the system to perform, an accurate and reliable measurement the automatic cleaning system must be introduced. There are no similar systems for railway measuring devices, but most similar are the one used in automotive industry. In April 2017 the international supplier Continental introduced a new camera cleaning system where the cleansing water is directed at the camera lenses, where autonomous cleaning process is comfortable and safe. Google subsidiary "Waymo" also developed a self-cleaning mode for their sensors. Here the dome covering the lidar kit that sits atop the car is cleaned with water and small windshield wipers. The wipers emerge and spin around the dome until it is freed from the dirt. In 2015, Ficosa has launched a solution to clean the lenses of sensors and cameras integrated in the car automatically. Named Sensor \& Camera Cleaning and patented by the company, this product removes dirt from cameras and sensors through a hybrid system that expels water and/or pressurized air depending on the need and lenses position, guaranteeing a proper performance in all operating conditions.

The stereo camera pair and a laser beam (pattern) system (Figure 8) can be used to create 3D data (point cloud) that enables precise measurement of the transversal position of the wheel in relation to the rail. 


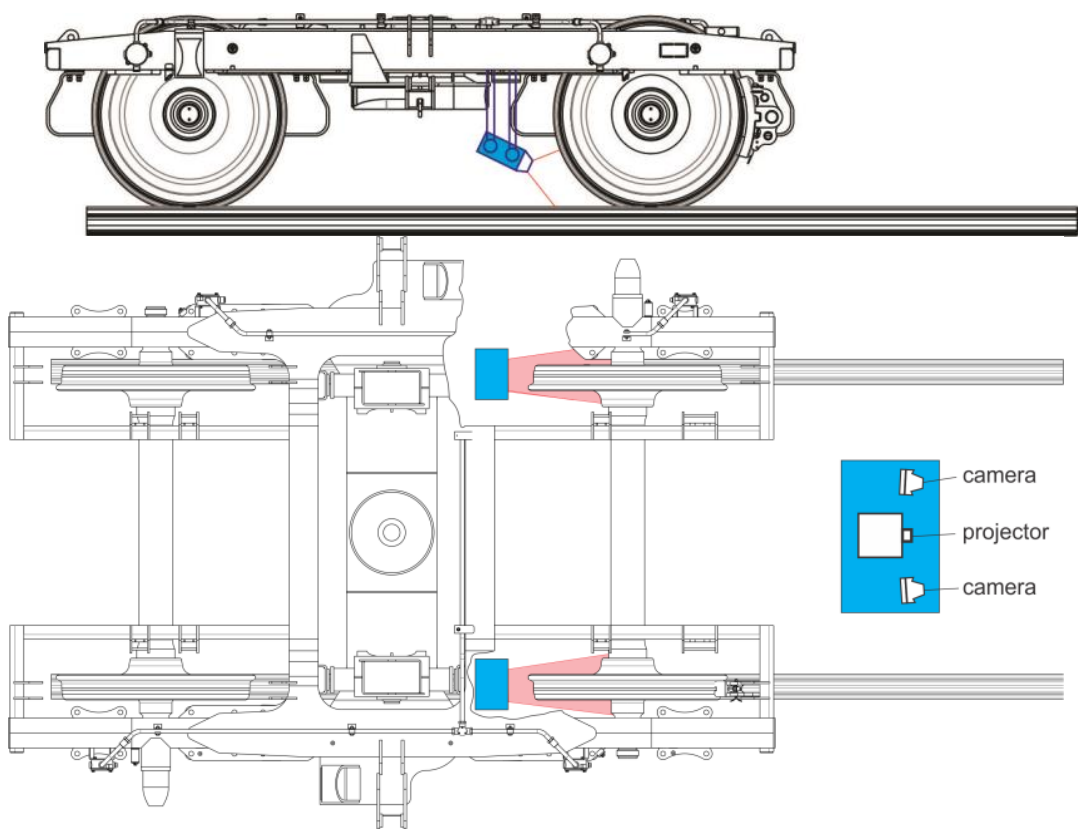

Fig. 8 Proposed measuring system

High-speed cameras with up to 5000 FPS will enable system functioning even at speeds greater than $200 \mathrm{~km} / \mathrm{h}$. At the highest speed of $200 \mathrm{~km} / \mathrm{h} \approx 55.6 \mathrm{~m} / \mathrm{s}$ measuring system can record position of the wheel in relation to the rail every $0.011 \mathrm{~m}$. Stereo camera pair enables continuous reliable point cloud reconstruction.

The usage of stereo camera pair can also enable measuring system to detect cracks and deformations of both rail track and wheel by comparing the current with historical pictures of the surface (undamaged condition). For continuous reliable measurement in all conditions, the housing needs to have minimum of IP 65 rating and whole measuring system should be insensitive to dust, rain, snow and waste along the track, while a specialized cleaning system for the protective glass should be developed (inspired by the camera and lidar cleaning systems for automotive industry that uses cleansing water directed trough jets directly to protective glass that covers camera lenses). Pressurized air and linear wipers should be considered for the quick drying.

Digital images of a contact line of a wheel and a track should be taken at a distance of $10 \mathrm{~cm}$, while framerate needs to be controlled by a rotary encoder. Thanks to the pathdependent image recording and analysis, each measurement can be assigned a clear position on the track and on the wheel. The resulting images must be analysed and processed using a beyond state of the art image processing algorithms to reliably measure the transversal position of the wheel in relation to the rail and to create a $3 \mathrm{D}$ reconstruction of the track and wheel profiles. The proposed system will allow high measuring speed at a short measuring distance, high measurement resolution and certainty, high recognition performance and low maintenance. 


\section{CONCLUSION}

The proposed solution has several clear advances compared to the already available solutions, like precise measurement of transversal position of the wheel in relation to the rail with an on-board device; low power consumption; self-sustainability; identification of the wheel and rail defects by one device and auto-cleaning system that makes measurement insensitive to dust, rain, snow and waste along the track.

So far there are no commercial solutions used for the continuous monitoring of transversal position of the wheel in relation to the rail other than DTK Wheel-Rail contact measurement system. However, the proposed solution has many advantages, like higher precision, lower power consumption, identification of wheel and rail defects and operability in hard condition (rain, snow, dust, ice) etc. The precision of proposed measuring device is high due to high speed stereo cameras. The algorithms for triangulation can be improved and adjusted to fulfil high performance demands and to enable adequate accuracy and reliability of measurement.

Stereo camera pair should be focused on recording the contact of the wheel and the rail, but in acquired videos and reconstructed point cloud cracks and other irregularities will be visible and a system for fault detection of both rail and wheel can be integrated into the proposed solution.

Acknowledgement: The paper is a part of the research done within the project "Research and development of new generation machine systems in the function of the technological development of Serbia" funded by the Faculty of Mechanical Engineering, University of Niš.

\section{REFERENCES}

[1] Matsumoto, A., Sato, Y., Ohno, H., Tomeoka, M., Matsumoto, K., Kurihara, J., Ogino, T., Tanimoto, M., Kishimoto, Y., Sato, Y. and Nakai, T., 2008. A new measuring method of wheel-rail contact forces and related considerations. Wear, 265(9-10), pp.1518-1525.

[2] Matsumoto, A., Sato, Y., Ohno, H., Shimizu, M., Kurihara, J., Tomeoka, M., Saitou, T., Michitsuji, Y., Tanimoto, M., Sato, Y. and Mizuno, M., 2012. Continuous observation of wheel/rail contact forces in curved track and theoretical considerations. Vehicle system dynamics, 50(sup1), pp.349-364.

[3] Zhu, T., Xiao, S., Yang, G., Ma, W. And Zhang, Z., 2014. An inverse dynamics method for railway vehicle systems. Transport, 29(1), pp.107-114.

[4] Wei, L., Zeng, J., Wu, P. And Gao, H., 2014. Indirect method for wheel-rail force measurement and derailment evaluation. Vehicle System Dynamics, 52(12), pp.1622-1641.

[5] Bagheri, V.R., Tehrani, P.H. and Younesian, D., 2017. Optimal strain gauge placement in instrumented wheelset for measuring wheel-rail contact forces. International Journal of Precision Engineering and Manufacturing, 18(11), pp.1519-1527.

[6] Milković, D., Simić, G., Jakovljević, Ž., Tanasković, J. and Lučanin, V., 2013. Wayside system for wheel-rail contact forces measurements. Measurement, 46(9), pp.3308-3318.

[7] DANOBATGROUP 2018, DW DANOBAT Wheel Profile Measuring, https://railways.danobatgroup. com/media/uploads/solutions/wheelset-wheel-profile-measuring-railways-dwpm111.pdf, viewed April 2018

[8] DEUTZER Tehnische Kohle 2018, Wheel-Rail contact measurement, http://www.deutzer.de/en/servicesen/strassenbahn-en/333-rsmstrassenbahn-en, viewed April 2018

[9] BVSYS 2018a, 3rdRailPositionCheck, http://www.bvsys.de/index.php/products_2/railway-inspectionsystems_6/39-3rdrailpositioncheck, viewed April 2018

[10] BVSYS 2018b, ProfileCheck, http://www.bvsys.de/index.php/products_2/railway-inspection-systems_6/13profilecheck, viewed April 2018 
[11] Mandelbaum, R., McDowell, L., Bogoni, L., Reich, B. and Hansen, M., 1998, October. Real-time stereo processing, obstacle detection, and terrain estimation from vehicle-mounted stereo cameras. In Applications of Computer Vision, 1998. WACV'98. Proceedings., Fourth IEEE Workshop on (pp. 288-289).

[12] Dineesh Mohan, A. Ranjith Ram, 2015, A Review on Depth Estimation for Computer Vision Applications, International Journal of Engineering and Innovative Technology (IJEIT) Volume 4, Issue 11

[13] Kovačić, K., Ivanjko, E. and Gold, H., 2013. Computer vision systems in road vehicles: a review. arXiv preprint arXiv:1310.0315.

[14] Stefan Heist, Peter Lutzke, Ingo Schmidt, Patrick Dietrich, Peter Kühmstedt, Andreas Tünnermann, Gunther Notni, High-speed three-dimensional shape measurement using GOBO projection, Optics and Lasers in Engineering, Vol. 87, 2016, pp. 90-96, ISSN 0143-8166,

[15] Tiago L. F. da Costa Pinto, Armando Albertazzi, 2014, 3D Active Stereo Measurement in a Regular Mesh wth Random Pattern and Laser Speckle Projection, ABCM Symposium Series in Mechatronics Vol. 6, pp. 281-289

[16] Yang, G., Sun, C., Wang, P. and Xu, Y., 2014. High-speed scanning stroboscopic fringe-pattern projection technology for three-dimensional shape precision measurement. Applied optics, 53(2), pp.174-183.

[17] Zuo, C., Chen, Q., Gu, G., Feng, S. and Feng, F., 2012. High-speed three-dimensional profilometry for multiple objects with complex shapes. Optics express, 20(17), pp.19493-19510.

[18] Schaffer, M., Grosse, M. and Kowarschik, R., 2010. High-speed pattern projection for three-dimensional shape measurement using laser speckles. Applied optics, 49(18), pp.3622-3629.

[19] Heist, S., Lutzke, P., Schmidt, I., Dietrich, P., Kühmstedt, P., Tünnermann, A. and Notni, G., 2016. Highspeed three-dimensional shape measurement using GOBO projection. Optics and Lasers in Engineering, 87, pp.90-96. 